\title{
Evaluation of Thick-Slab Overlapping MIP Images of Contrast- Enhanced 3D T1-Weighted CUBE for Detection of Intracranial Metastases: A Pilot Study for Comparison of Lesion Detection, Interpretation Time, and Sensitivity with Nonoverlapping CUBE MIP, CUBE, and Inversion-Recovery-Prepared Fast-Spoiled Gradient Recalled Brain Volume
}

\author{
(D)B.C. Yoon, (D)A.F. Saad, (DP. Rezaii, (D) M. Wintermark, (D) G. Zaharchuk, and (D) M. Iv
}

\begin{abstract}
BACKGROUND AND PURPOSE: Early and accurate identification of cerebral metastases is important for prognostication and treatment planning although this process is often time consuming and labor intensive, especially with the hundreds of images associated with 3D volumetric imaging. This study aimed to evaluate the benefits of thick-slab overlapping MIPs constructed from contrast-enhanced T7-weighted CUBE (overlapping CUBE MIP) for the detection of brain metastases in comparison with traditional CUBE and inversionrecovery prepared fast-spoiled gradient recalled brain volume (IR-FSPGR-BRAVO) and nonoverlapping CUBE MIP.
\end{abstract}

MATERIALS AND METHODS: A retrospective review of 48 patients with cerebral metastases was performed at our institution from June 2016 to October 2017. Brain MRIs, which were acquired on multiple 3T scanners, included gadolinium-enhanced T7-weighted IR-FSPGRBRAVO and CUBE, with subsequent generation of nonoverlapping CUBE MIP and overlapping CUBE MIP. Two blinded radiologists identified the total number and location of metastases on each image type. The Cohen $\kappa$ was used to determine interrater agreement. Sensitivity, interpretation time, and lesion contrast-to-noise ratio were assessed.

RESULTS: Interrater agreement for identification of metastases was fair-to-moderate for all image types $(\kappa=0.222-0.598)$. The total number of metastases identified was not significantly different across the image types. Interpretation time for CUBE MIPs was significantly shorter than for CUBE and IR-FSPGR-BRAVO, saving at least 50 seconds per case on average $(P<.001)$. The mean lesion contrast-to-noise ratio for both CUBE MIPs was higher than for IR-FSPGR-BRAVO. The mean contrast-to-noise ratio for small lesions ( $<4 \mathrm{~mm}$ ) was lower for nonoverlapping CUBE MIP (1.55) than for overlapping CUBE MIP (2.35). For both readers, the sensitivity for lesion detection was high for all image types but highest for overlapping CUBE MIP and CUBE (0.93-0.97).

CONCLUSIONS: This study suggests that the use of overlapping CUBE MIP or nonoverlapping CUBE MIP for the detection of brain metastases can reduce interpretation time without sacrificing sensitivity, though the contrast-to-noise ratio of lesions is highest for overlapping CUBE MIP.

ABBREVIATIONS: CNR = contrast-to-noise ratio; IR-FSPGR-BRAVO = inversion-recovery-prepared fast-spoiled gradient recalled brain volume; $\mathrm{nC}$-MIP $=$ nonoverlapping CUBE MIP; OC-MIP = overlapping CUBE MIP; SRS = stereotactic radiosurgery; XR = cross-reference

T he early and accurate identification of brain metastases in patients with systemic cancers has important implications for patient prognosis and treatment strategy because a greater number of lesions at presentation correlates with decreased survival. ${ }^{1}$ In patients with a small number of metastases, surgical resection or stereotactic radiosurgery (SRS) may be pursued, while whole-

Received September 23, 2017; accepted after revision June 16, 2018.

From the Department of Radiology, Division of Neuroimaging and Neurointervention, Stanford University, Stanford, California.

Paper previously presented as an electronic exhibit at: Annual Meeting of the American Society of Neuroradiology and the Foundation of the ASNR Symposium, April 22-27, 2017; Long Beach, California (\#eP-50).

Please address correspondence to Michael Iv, MD, Department of Radiology, Division of Neuroimaging and Neurointervention, Stanford University, 300 Pasteur Dr, Grant Building, Room S031E, Stanford, CA 94305; e-mail: miv@stanford.edu, @Michael_Iv_MD

http://dx.doi.org/10.3174/ajnr.A5747 brain radiation therapy is generally recommended for those with an extensive lesion burden. ${ }^{2,3}$ Previous studies have shown that SRS alone is an effective treatment and provides good local tumor control in patients with up to 10 brain metastases. ${ }^{4,5}$ Additionally, the rate of local control with SRS is greater for small lesions, which further stresses the importance of early detection. The choice of SRS or whole-brain radiation therapy for the treatment of brain metastases in individuals with a specific number of lesions is important because the risk of radiation-induced dementia and neurocognitive decline associated with whole-brain radiation therapy can be potentially avoided with SRS.

Counting multiple small metastases is often laborious and time-consuming, especially with the hundreds of images associated with $3 \mathrm{D}$ volumetric imaging. Multiple investigators have demonstrated improved lesion detection using 3D T1-weighted volumetric fast spin-echo sequences (CUBE, GE Healthcare, Mil- 
waukee, Wisconsin; sampling perfection with application optimized contrasts by using different flip angle evolution [SPACE], Siemens, Erlangen, Germany; volume isotropic turbo spin-echo [VISTA], Philips Healthcare, Best, the Netherlands). ${ }^{6-13}$ CUBE (and similar sequences) uses a variable flip angle technique and higher echo-train length to acquire gap-free volumetric images with reduced acquisition time and specific absorption rate. ${ }^{7}$ Furthermore, the black-blood properties offered by CUBE allow good background vascular suppression and provide a higher contrast-tonoise ratio (CNR) than 3D T1-weighted gradient-echo sequences (inversion-recovery-prepared fast spoiled gradient recalled brain volume [IR-FSPGR-BRAVO], GE Healthcare; MPRAGE, Siemens; 3D TFE, Phillips Healthcare). ${ }^{7}$

The use of maximum-intensity-projection images has become standard in chest imaging when evaluating pulmonary nodules because MIPs have been shown to enhance lesion detection and reduce the total amount of time the radiologist spends searching for small nodules. ${ }^{14-17}$ In neuroimaging, 3D volumetric fast spinecho imaging is ideally suited to MIP reconstruction because of its black-blood and increased CNR properties. However, the use of MIP images in the detection of brain metastases has been limited. ${ }^{18,19}$ Recently, Bae et al ${ }^{18}$ have shown that nonoverlapping 5-mm-thick MIPs of 3D T1-weighted turbo spin-echo significantly reduced interpretation time without sacrificing diagnostic accuracy but with an increased false-positive rate, compared with the source 1-mm images. Thick-section MIPs reconstructed with slice overlapping can potentially reduce artifacts from partial volume averaging and improve visualization of lesions. Therefore, in this study, we examined whether the use of thick-slab overlapping MIPs constructed from gadolinium-enhanced 3D T1-weighted CUBE (overlapping CUBE MIP [oC-MIP]) would allow improved visualization and quicker and more sensitive detection of brain metastases compared with nonoverlapping CUBE MIP (nC-MIP) as well as source 3D T1weighted CUBE and IR-FSPGR-BRAVO.

\section{MATERIALS AND METHODS}

We performed an institutional review board-approved retrospective study of patients with brain metastases who had MRIs from June 2016 to October 2017 at a single academic institution. Patients with a diagnosis of metastatic disease from any primary cancer who had brain MR imaging during the study period were identified through our PACS imaging data base by keyword search criteria. The specific keywords used were "metastasis" and "metastases." On the basis of finalized reports associated with the MRIs, patients with at least 2 intra-axial brain metastases were included in the study.

\section{MR Imaging Protocol}

All brain MRIs were performed on multiple 3T scanners (Discovery 750; GE Healthcare) using our institutional brain metastasis protocol, which consisted of precontrast and postcontrast images, the latter acquired following the intravenous administration of $0.1 \mathrm{mmol} / \mathrm{kg}$ of gadobenate dimeglumine (MultiHance; Bracco Diagnostics, Princeton, New Jersey). Gadobenate dimeglumine is the specific contrast agent used at our institution because of its high relaxivity, which allows improved lesion conspicuity.
Postgadolinium sequences were obtained in the following order: sagittal 3D T1-weighted CUBE $(\mathrm{TR}=600 \mathrm{~ms}$, TE $=13 \mathrm{~ms}$, slice thickness $=1 \mathrm{~mm}$, echo-train length $=28$, flip angle $=90^{\circ}$, matrix $=256 \times 256 \mathrm{~mm}, \mathrm{FOV}=250 \mathrm{~mm}$, in-plane resolution $=$ $0.977 \mathrm{~mm}$, bandwidth $=244 \mathrm{~Hz}$, number of averages $=1$, imaging time $=2$ minutes 15 seconds) with $1-\mathrm{mm}$ axial and coronal reformats and axial 3D T1-weighted IR-FSPGR-BRAVO (TR = $9.2 \mathrm{~ms}, \mathrm{TE}=3.7 \mathrm{~ms}$, TI $=400 \mathrm{~ms}$, slice thickness $=1 \mathrm{~mm}$, echo-train length $=1$, flip angle $=13^{\circ}$, matrix $=256 \times 256 \mathrm{~mm}$, $\mathrm{FOV}=240 \mathrm{~mm}$, in-plane resolution $=0.938 \mathrm{~mm}$, bandwidth $=$ $195 \mathrm{~Hz}$, number of averages $=1$, imaging time $=3$ minutes 30 seconds) with 1-mm sagittal and coronal reformats. IR-FSPGRBRAVO was the last sequence performed in our protocol because increased scan time delay after contrast administration allows improved lesion detection.

Axial MIP images (oC-MIP: 10-mm sections reconstructed at 4-mm intervals; $\mathrm{nC}$-MIP: 5-mm sections at 5-mm intervals) were then constructed from the contrast-enhanced T1-weighted CUBE images. Image parameters for the nC-MIPs were chosen on the basis of results from a recent prior study showing their benefits for the detection of brain metastases. ${ }^{18}$ Image parameters for the oCMIPs were chosen on the basis of our clinical experience of using thick-slabbed MIPs for detection of enhancing brain lesions because no prior studies, to our knowledge, have reported optimal values for overlapping MIPs. Axial MIPs of IR-FSPGR-BRAVO (10-mm sections reconstructed at 4-mm intervals) were also generated solely for comparison of CNR with CUBE MIPs. These specific MIPS were not used for detection of metastases in this study because we and others have found them to be of little clinical utility, in large part due to the difficulty in distinguishing true enhancing metastases from excessive background vascular enhancement. ${ }^{18}$

\section{Determination of Ground Truth for Brain Metastases}

A neuroradiologist with $>10$ years of experience who did not participate in counting of the metastases as part of the study carefully reviewed all sequences from the initial MR imaging, including precontrast (diffusion-weighted imaging, gradient recalled, T2-weighted fast spin-echo, 3D T1 CUBE) and postcontrast (3D T1 CUBE, IR-FSPGR-BRAVO, 3D CUBE FLAIR) images as well as any follow-up MRIs that were acquired before any intervening treatment. A brain metastasis was positively identified and recorded (image slice number of the lesion and its anatomic location) if the following criteria were present: a nonvascular enhancing lesion with or without associated FLAIR or diffusion signal intensity, surrounding edema, or hemorrhage that was unchanged or increased in size on follow-up MR imaging. For this study, any postsurgical enhancement (defined as enhancement contiguous with a remote surgical resection cavity) and leptomeningeal enhancement were excluded.

\section{Image Analysis}

A neuroradiologist with 10 years of experience (reader 1) and a radiology resident with 2 years of experience (reader 2) counted the number of brain metastases for each patient during 4 sessions separated by 1 week between each session. Each reader independently evaluated CUBE in 1 session, $\mathrm{nC}-\mathrm{MIP}$ alone in the second 
session, oC-MIP alone in the third session, and IR-FSPGRBRAVO in the last session. In additional sessions separated by 1 week, each reader also independently evaluated nC-MIP and oC-MIP with the option to cross-reference a lesion with source images to confirm the authenticity of a questionable lesion (nC$\mathrm{MIP}+$ cross-reference $[\mathrm{XR}]$ and oC-MIP $+\mathrm{XR}$, respectively); this was performed to determine whether the simultaneous use of cross-referencing affected interpretation time and detection sensitivity. The order of patients during each session was randomized. The number of metastases identified and the time for each reader to identify and count all lesions (time for interpretation) were recorded. During the counting process, readers also recorded the image slice number and anatomic location of each lesion on each sequence using speech recognition software $(\mathrm{Nu}-$ ance PowerScribe 360 Reporting; Nuance Communications, Burlington, Massachusetts).

The contrast-to-noise ratio was calculated using the smallest $(<4 \mathrm{~mm})$ and largest metastatic lesions identified on CUBE, both CUBE MIPs, IR-FSPGR-BRAVO, and IR-FSPGR-BRAVO MIPs with the following formula: Mean Signal Intensity of Lesion Mean Signal Intensity of Normal White Matter / SD of Lesion Signal Intensity. ${ }^{8}$

\section{Statistical Analysis}

Due to the lack of histopathologic data, our study did not have an absolute standard of truth. Our ground truth was based on the evaluation of all sequences on the initial MR imaging and comparison of lesions with any follow-up MR imaging in which there was no intervening treatment. Therefore, we aimed to compare the sensitivities and discrepancy rates of all image types rather than to determine diagnostic accuracy; this method has been similarly used in previous analyses of pulmonary nodules. ${ }^{20}$

Interrater agreement for identification of metastases was calculated using the Cohen $\kappa$ coefficient with the following interpretation model of $\kappa$ : 0-.20, slight; 0.21-0.40, fair; 0.41-0.60, moderate; 0.61-0.80, substantial; and 0.81-1, almost perfect. $^{21}$ Specifically, of the total pool of ground truth metastases, agreement for the presence of each individual lesion was assessed as follows: 1) both readers agreed that a lesion was present, 2) reader 1 identified a lesion but reader 2 did not, 3 ) reader 2 identified a lesion but reader 1 did not, and 4) both readers agreed that a lesion was not present. The total number of lesions detected on each image type and time for interpretation were compared between readers using 1-way analysis of variance with a Bonferroni adjustment. A $P<.013$ (calculated by .05/4) was considered statistically significant in comparing the 4 image types (CUBE, nC-MIP, oC-MIP, IR-FSPGR-BRAVO), as determined by the Bonferroni adjustment. ${ }^{22}$ The CNR of the smallest and largest lesions on each series was reported as mean $\pm \mathrm{SD}$. In a subgroup analysis, the time for interpretation was compared between all CUBE MIPs (nC-MIP, oC-MIP, nC-MIP+XR, oC$\mathrm{MIP}+\mathrm{XR}$ ) and source CUBE, using 1-way analysis of variance with a Bonferroni adjustment. A $P<.01$ (calculated by .05/5) was considered statistically significant in comparing these 5 image types, as determined by the Bonferroni adjustment. ${ }^{22}$ Lesion-detection sensitivity, mean false-negative, mean falsepositive, and mean discrepancy (total number of false-posi-
Table 1: Interrater agreement using the Cohen $\kappa$ coefficient for T1-weighted CUBE, nC-MIP, oC-MIP, and IR-FSPGR-BRAVO

\begin{tabular}{lccl}
\hline \multicolumn{1}{c}{ Image Type } & $\boldsymbol{\kappa}$ & $\begin{array}{c}\mathbf{9 5 \%} \\
\text { Confidence } \\
\text { Interval }\end{array}$ & Agreement \\
\hline CUBE & 0.235 & $0.024-0.447$ & Fair \\
nC-MIP & 0.222 & $0.069-0.374$ & Fair \\
OC-MIP & 0.598 & $0.371-0.825$ & Moderate \\
IR-FSPGR-BRAVO & 0.445 & $0.290-0.599$ & Moderate \\
\hline
\end{tabular}

tives and false-negatives) per case were assessed for all image analysis types.

\section{RESULTS}

A total of 308 metastases were identified in 48 patients, consisting of 37 women and 11 men (mean age: $62.4 \pm 13$ years; age range, 29-81 years). Twenty-six patients had primary non-small cell lung cancer, 1 had small cell lung cancer, 13 had breast cancer, 2 had melanoma, 2 had renal cell carcinoma, 1 had esophageal cancer, and 2 had ovarian cancer. One patient had a history of LiFraumeni syndrome and had multiple primary cancers, including colon, lung, breast, uterine, and hepatocellular carcinoma. Of the 48 patients, 27 had undergone prior treatment for brain metastases, including surgical resection, whole-brain radiation therapy, and/or SRS.

Interrater agreement for the presence of individual metastatic lesions was fair-to-moderate across all image types $(\kappa=0.222$ $0.598)$ but highest for oC-MIP $(\kappa=0.598)$ (Table 1$)$. No significant difference was found among the total number of metastases on any of the 4 main image types $(P=.062)$ : CUBE (319 for reader one, 336 for reader 2 ), $\mathrm{nC}$-MIP ( 325 for reader one, 313 for reader 2), oC-MIP (327 for reader one, 333 for reader 2), IRFSPGR-BRAVO (289 for reader one, 303 for reader 2) (Fig 1).

A significant difference in the time for interpretation among the image types was found for both readers $(P<.001)$ (Fig $2 A)$. The time for interpretation using oC-MIP (mean: $55.2 \pm 25.1$ seconds for reader one, $94.7 \pm 36.5$ seconds for reader 2 ) and nC-MIP (58.2 \pm 32.9 seconds for reader one, $97.0 \pm 23.4$ seconds for reader 2) was significantly reduced compared with CUBE $(109.8 \pm 47.5$ seconds for reader one, $P<.001 ; 173.5 \pm 67.7$ seconds for reader $2, P<.001)$ and IR-FSPGR-BRAVO $(124.6 \pm$ 48 seconds for reader one, $P<.001 ; 195 \pm 64.8$ seconds for reader $2, P<.001$ ), with a savings of at least 50 seconds per case (on average). In a subgroup analysis of all CUBE image types, time for interpretation using oC-MIP $+\mathrm{XR}(61.9 \pm 27.7$ seconds for reader one, $91.7 \pm 35.0$ seconds for reader 2$)$ and $\mathrm{nC}-\mathrm{MIP}+\mathrm{XR}$ $(71.7 \pm 32.0$ seconds for reader one, $94.3 \pm 33.0$ seconds for reader 2$)$ was significantly reduced compared with CUBE $(P<$ .001) (Fig 2B). However, time for interpretation was not significantly different among oC-MIP, nC-MIP, oC-MIP +XR, and nC-MIP+XR.

The conspicuity of the lesions as indicated by CNR on the 4 image types also differed for both small $(<4 \mathrm{~mm})$ and large lesions (Table 2 and Fig 3 ). For both lesion groups, the mean CNR was the highest for oC-MIP and higher with CUBE and both CUBE MIPs than with IR-FSPGR-BRAVO and IR-FSPGRBRAVO MIP. The mean CNR for small lesions was lower with nC-MIP $(1.55 \pm 0.3)$ than with CUBE $(2.35 \pm 2.0)$ or oC-MIP $(2.35 \pm 1.64)$. 


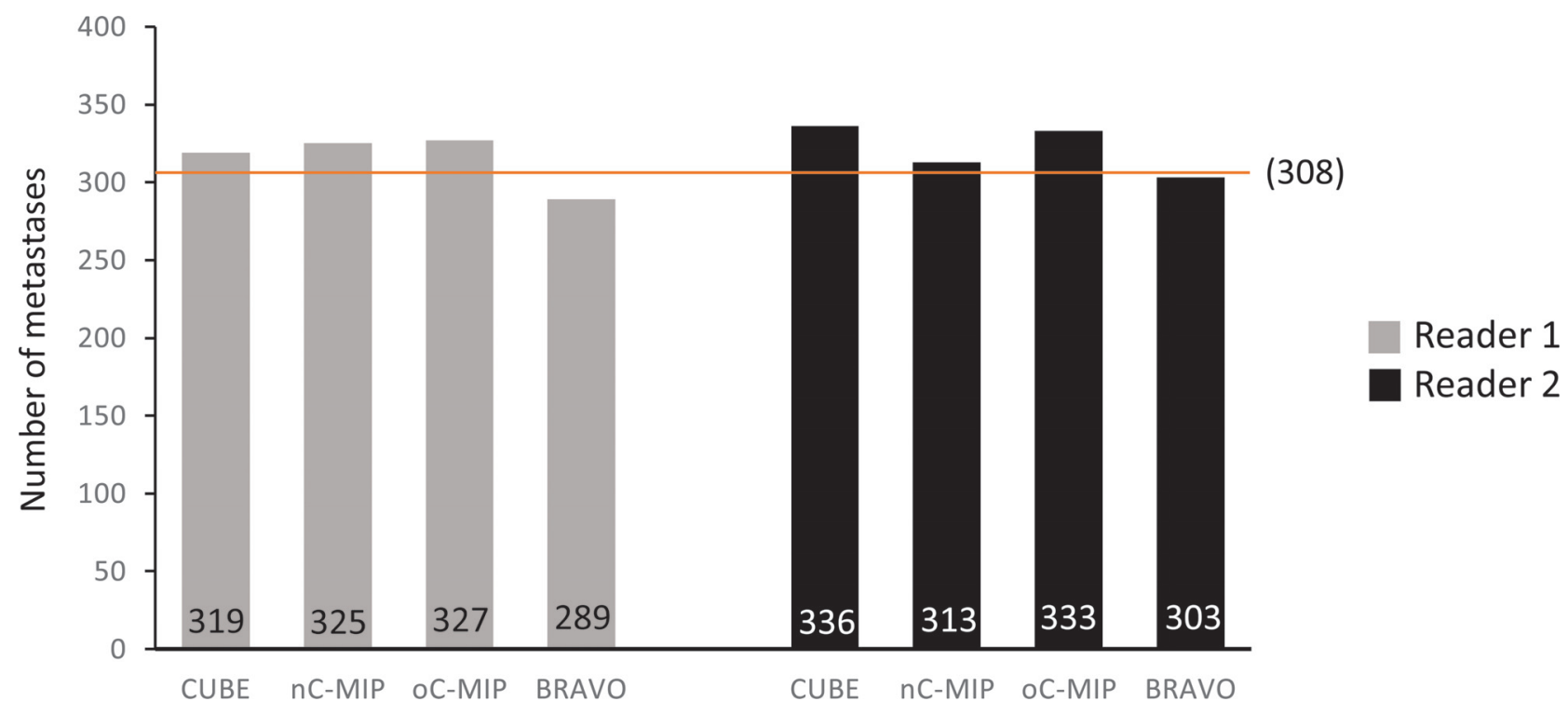

FIG 1. Total number of brain metastases detected. No significant difference was found among the total number of metastases detected using CUBE, nC-MIP, OC-MIP, and IR-FSPGR-BRAVO $(P=.062)$ using l-way ANOVA with a Bonferroni adjustment. The orange line denotes the total number of ground truth lesions $(n=308)$.

For both readers, the sensitivity for lesion detection was high for all image types. Of CUBE, nC-MIP, oC-MIP, and IR-FSPGRBRAVO, sensitivity was highest for oC-MIP (0.96 for both readers) and CUBE (0.97 for reader one, 0.93 for reader 2) (Table 3). Sensitivity was slightly lower for nC-MIP (0.95 for reader one, 0.90 for reader 2) and even lower for IR-FSPGR-BRAVO (0.92 for reader one, 0.89 for reader 2 ). On average, there was $<1$ falsenegative and 1 false-positive case per patient across all image types (Table 3). The mean discrepancy rate (total number of false-negative and false-positive lesions per case) was also $<1$ lesion per patient on all image types except with nC-MIP and IR-FSPGRBRAVO for reader 2. Mean false-positives per case were reduced with oC-MIP + XR (0.21 for reader one, 0.23 for reader 2$)$ compared with oC-MIP (0.54 for reader one, 0.33 for reader 2$)$ and with $\mathrm{nC}-\mathrm{MIP}+\mathrm{XR}(0.25$ for reader one, 0.29 for reader 2$)$ compared with nC-MIP ( 0.58 for reader one, 0.71 for reader 2$)$.

\section{DISCUSSION}

In this study, we found that the use of oC-MIP or nC-MIP reduced interpretation time without sacrificing lesion detection sensitivity compared with traditional CUBE and IR-FSPGRBRAVO. The coupling of CUBE MIPs with the option to crossreference a questionable lesion to source images further reduced false-positives without significantly changing the time for interpretation compared with MIPs alone. The CNR of brain metastases was higher with CUBE and both CUBE MIPs than with IRFSPGR-BRAVO and IR-FSPGR-BRAVO MIP, though the CNR of small and large metastases was highest with thick-slab oC-MIP. Interrater agreement for the detection of brain metastases was fair-to-moderate across all image types but highest with oC-MIP. The findings in our study are consistent with those of other investigators regarding enhanced lesion detection and increased CNR using $3 \mathrm{D}$ fast spin-echo imaging compared with gradient-echo imaging. ${ }^{7,8}$ CUBE images are ideally suited for MIP reconstruction because there is an inherent reduction in the amount of background vascular enhancement, which further reduces background image noise and increases lesion conspicuity.

An issue of clinical importance for practicing radiologists that has received less research attention is interpretation time, a particularly relevant issue with the increasing use of $3 \mathrm{D}$ volumetric sequences. Tasks such as counting individual millimetric metastases is of high importance in patient management, but this remains time-consuming and laborious. A recent study demonstrated that the use of nonoverlapping 5-mm-thick MIP reformations of contrast-enhanced 3D T1-weighted turbo spin-echo imaging yielded a shorter time for interpretation with sensitivity comparable with that of the 1-mm source images. ${ }^{18}$ Similarly, we found that the use of CUBE MIPs resulted in significantly reduced interpretation times compared with both source CUBE and IRFSPGR-BRAVO, without a reduction in detection sensitivity. When we compared the 5-mm-thick nC-MIP (used in the study of Bae et $\mathrm{al}^{18}$ ) and 10-mm-thick oC-MIP, the sensitivity for detection of cerebral metastases was slightly higher with the latter. Moreover, while the mean discrepancy (number of missed lesions) was $<1$ lesion per case between the 2 MIPs for reader 1 , the mean discrepancy and the SD were slightly higher for reader 2, the radiology resident. This is not surprising because the CNR is dependent on the SNR, which increases with MIP technique and slice thickness. There is also less effect of partial volume averaging with overlapping than nonoverlapping MIPs, which contributes to increased lesion conspicuity and detection. As an example, Fig 4 demonstrates a small lesion that is visible over a greater number of slices on oC-MIP than on nC-MIP. There is also easier tracking of vessels on oC-MIP because vessels are seen continuously over multiple slices. Thus, the use of thick-slab oC-MIP can be a useful tool for less experienced radiologists, such as trainees, to detect cerebral metastases.

The absolute number of brain metastases identified on MR imaging is important in determining treatment planning (eg, selection of SRS or whole-brain radiation therapy). Our results 


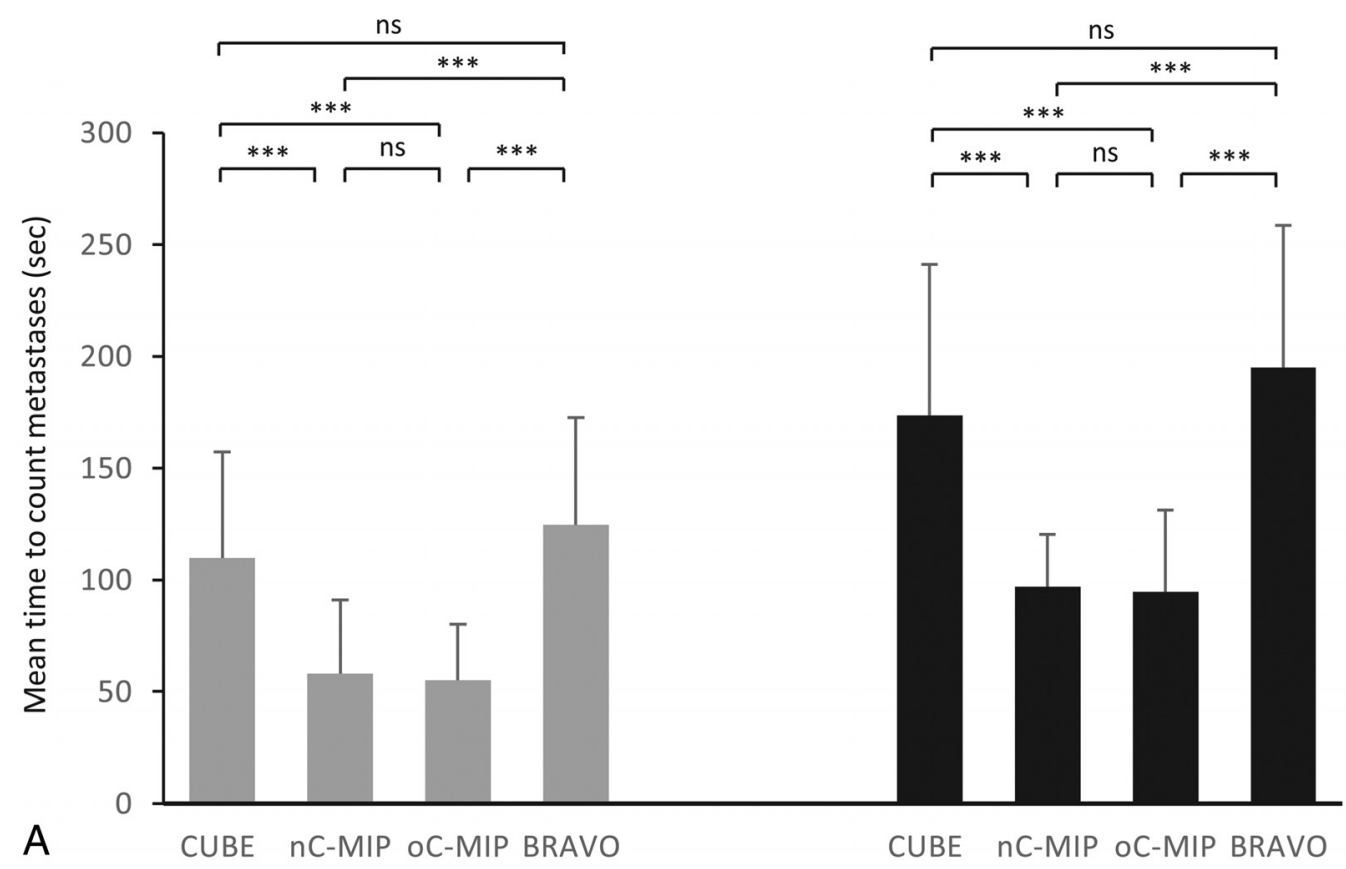

Reader 1

Reader 2

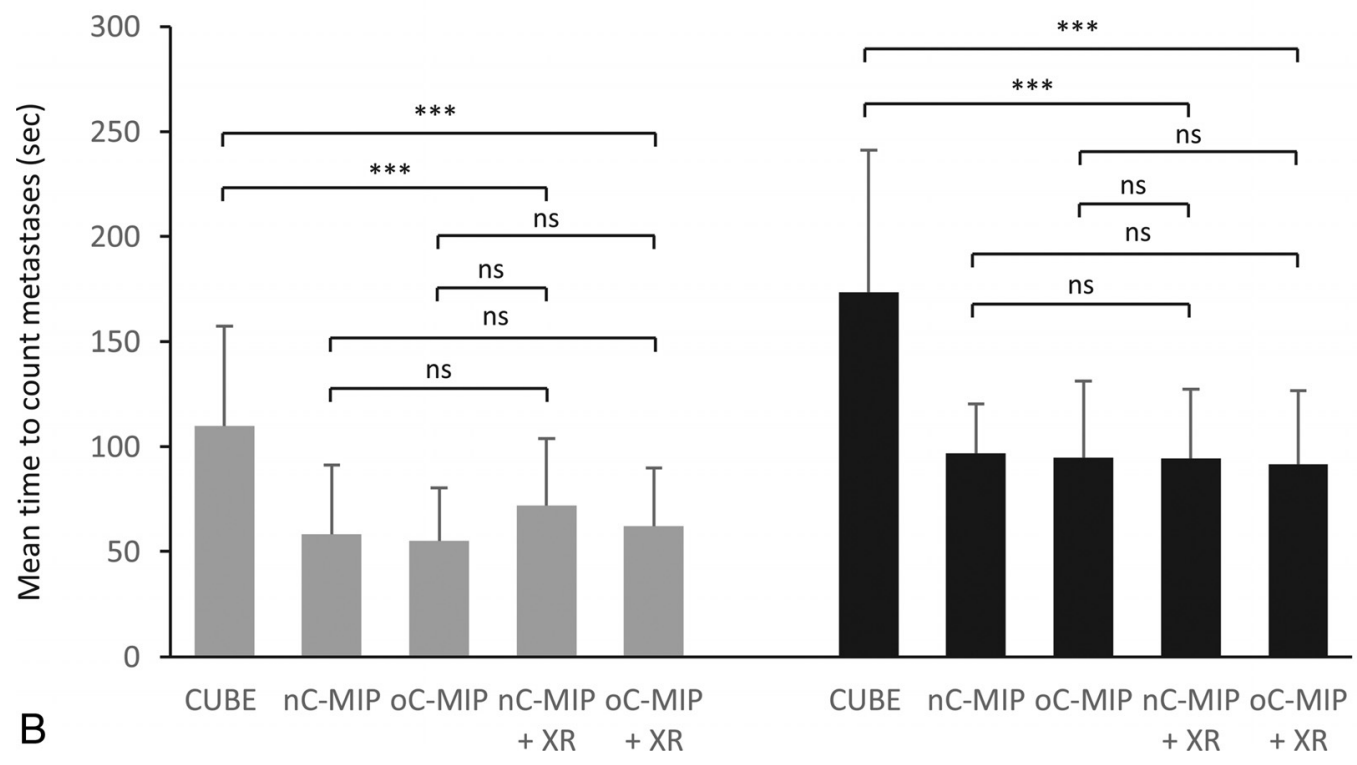

FIG 2. Mean time for interpretation. $A$, Both readers had significantly reduced interpretation time using nC-MIP and oC-MIP compared with CUBE and IR-FSPGR-BRAVO, saving at least 50 seconds per case (on average). B, The use of nC-MIP and oC-MIP with the option to crossreference an equivocal lesion to the source images ( $\mathrm{C} C-\mathrm{MIP}+\mathrm{XR}$ and $\mathrm{OC}-\mathrm{MIP}+\mathrm{XR}$, respectively) did not result in a significant change in interpretation time compared with the use of nC-MIP or OC-MIP alone. However, time for interpretation for all CUBE MIPs was significantly reduced compared with CUBE. Error bars represent the SD. One-way ANOVA with a Bonferroni adjustment. Triple asterisks indicate $P<.001$; ns, no significance.

Table 2: CNR of the smallest and largest metastases on CUBE, nC-MIP, OC-MIP, IR-FSPGR-BRAVO, and overlapping IR-FSPGRBRAVO MIP

\begin{tabular}{lcc}
\hline \multicolumn{1}{c}{ Image Type } & $\begin{array}{c}\text { CNR of } \\
\text { the Smallest } \\
\text { Lesion }(<4 \mathrm{~mm})\end{array}$ & $\begin{array}{c}\text { CNR of } \\
\text { the Largest } \\
\text { Lesion }\end{array}$ \\
\hline CUBE & $2.35 \pm 2.0$ & $2.49 \pm 1.84$ \\
nC-MIP & $1.55 \pm 0.53$ & $2.69 \pm 0.93$ \\
OC-MIP & $2.35 \pm 1.64$ & $3.23 \pm 1.92$ \\
IR-FSPGR-BRAVO & $0.62 \pm 0.39$ & $1.45 \pm 1.14$ \\
IR-FSPGR-BRAVO MIP & $0.90 \pm 0.78$ & $1.69 \pm 1.14$ \\
\hline
\end{tabular}

${ }^{\mathrm{a}}$ Numbers are means. indicate a small number of discrepant lesions per case using oC-MIP and CUBE, with both image types averaging $<1$ missed lesion per case in both readers. While not statistically significant, the total number of metastases detected with IR-FSPGR-BRAVO was also slightly less than with CUBE and both CUBE MIPs. This feature may have been related to IR-FSPGR-BRAVO failing to conspicuously demonstrate some lesions, which is likely due to the lower CNR associated with gradient-echo imaging (despite this sequence being the last acquired in our imaging protocol). Regarding lesion detection using MIPs, the discrepancy rate can be decreased by concurrently cross-referencing any lesions in question to the source images to confirm that they are true lesions. 

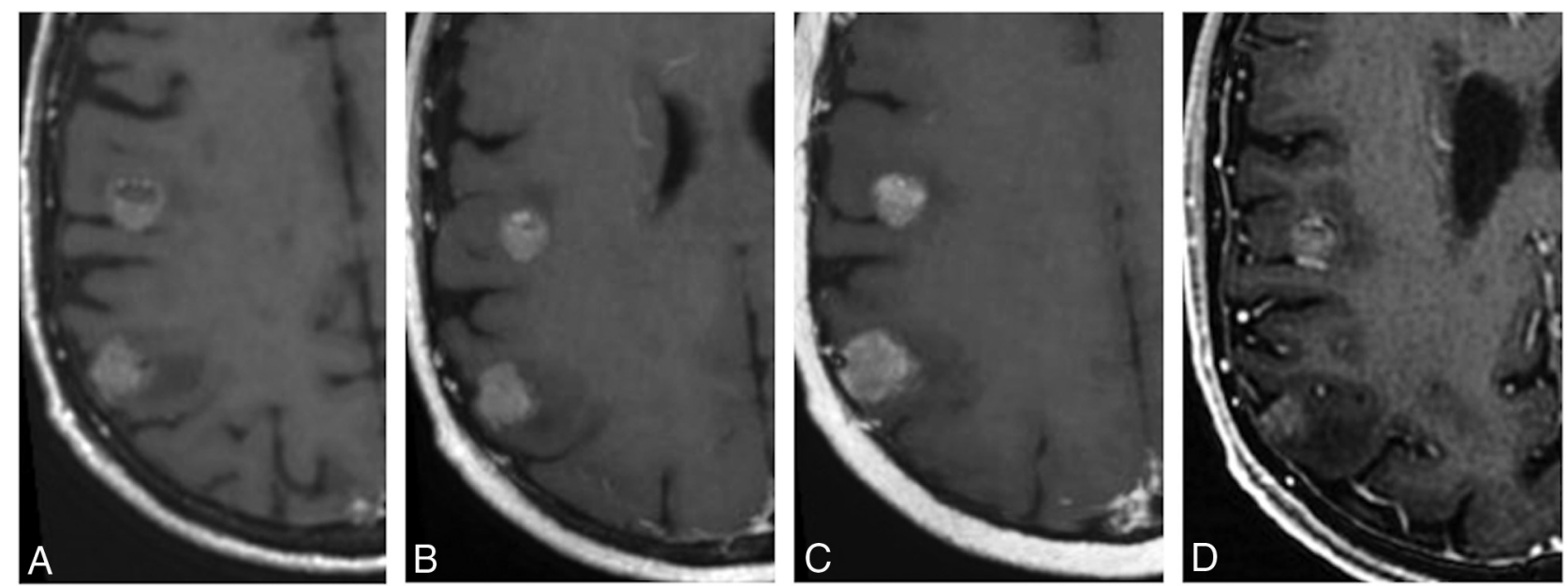

FIG 3. Enhancing cerebral metastases in a 74-year-old male with metastatic tongue squamous cell carcinoma. Postcontrast T1-weighted CUBE $(A)$, nonoverlapping CUBE MIP (nC-MIP) (B), overlapping CUBE MIP (OC-MIP) (C), and IR-FSPGR-BRAVO (D) images demonstrate enhancing metastatic lesions. The lesions appear most conspicuous with CUBE MIPs ( $B$ and $C)$. The contrast-to-noise ratio of lesions was highest for oC-MIP (C).

Table 3: Sensitivity, number of false-negatives, number of false-positives, and number of discrepant lesions (FN + FP) per case for CUBE, nC-MIP, oC-MIP, IR-FSPGR-BRAVO, and non-overlapping and overlapping CUBE MIPs ${ }^{\mathrm{a}}$

\begin{tabular}{|c|c|c|c|c|c|c|c|c|}
\hline & \multicolumn{4}{|c|}{ Reader 1} & \multicolumn{4}{|c|}{ Reader 2} \\
\hline & $\begin{array}{c}\% \\
\text { Sensitivity }\end{array}$ & Mean FN & Mean FP & $\begin{array}{c}\text { Mean } \\
\text { Discrepancy }\end{array}$ & $\begin{array}{c}\% \\
\text { Sensitivity }\end{array}$ & Mean FN & Mean FP & $\begin{array}{c}\text { Mean } \\
\text { Discrepancy }\end{array}$ \\
\hline CUBE & $97.1 \pm 14.8$ & $0.08 \pm 0.28$ & $0.31 \pm 0.63$ & $0.40 \pm 0.65$ & $93.0 \pm 16.8$ & $0.38 \pm 0.70$ & $0.38 \pm 0.82$ & $0.75 \pm 0.93$ \\
\hline nC-MIP & $94.7 \pm 20.4$ & $0.08 \pm 0.28$ & $0.58 \pm 0.98$ & $0.65 \pm 0.96$ & $90.2 \pm 16.8$ & $0.60 \pm 0.96$ & $0.71 \pm 1.98$ & $1.31 \pm 2.00$ \\
\hline OC-MIP & $95.8 \pm 15.4$ & $0.19 \pm 0.49$ & $0.54 \pm 0.92$ & $0.73 \pm 0.94$ & $95.8 \pm 15.4$ & $0.19 \pm 0.50$ & $0.33 \pm 0.69$ & $0.75 \pm 0.93$ \\
\hline BRAVO & $91.5 \pm 17.0$ & $0.58 \pm 0.99$ & $0.21 \pm 0.50$ & $0.79 \pm 0.99$ & $89.0 \pm 19.5$ & $0.85 \pm 1.49$ & $0.31 \pm 0.59$ & $1.17 \pm 1.42$ \\
\hline $\mathrm{nC}-\mathrm{MIP}+\mathrm{XR}$ & $95.5 \pm 9.8$ & $0.33 \pm 0.66$ & $0.25 \pm 0.70$ & $0.58 \pm 0.87$ & $91.2 \pm 12.3$ & $0.67 \pm 0.93$ & $0.29 \pm 0.74$ & $0.95 \pm 1.01$ \\
\hline$O C-M I P+X R$ & $96.6 \pm 7.3$ & $0.29 \pm 0.62$ & $0.21 \pm 0.46$ & $0.68 \pm 0.96$ & $94.8 \pm 12.3$ & $0.38 \pm 0.79$ & $0.23 \pm 0.47$ & $0.60 \pm 0.82$ \\
\hline
\end{tabular}

Note:-FN indicates false-negative; FP, false-positive.

${ }^{a}$ With the option to cross-reference a lesion to the source images (nC-MIP+XR and oC-MIP+XR, respectively) for both readers. Numbers are means.

OC-MIP
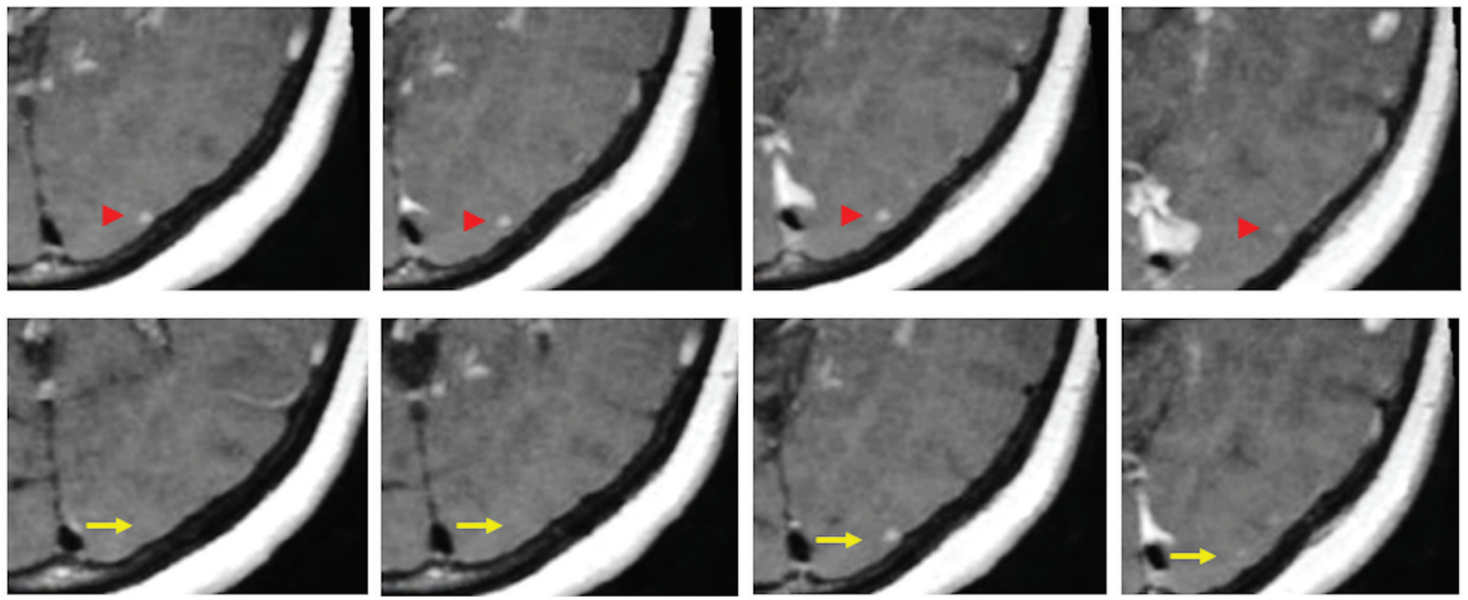

FIG 4. Comparison between nonoverlapping and overlapping CUBE MIP. A small 3-mm metastasis in a 57-year-old woman with non-small cell lung cancer is seen across 4 different slices with oC-MIP but is only identified on 2 slices with nC-MIP.

While missing a few metastatic lesions may not mean much in an individual with innumerable lesions, it may potentially change management in patients with fewer lesions who are being considered for SRS because certain institutions may only offer SRS to patients with up to a specific number of lesions.

In chest imaging, MIPs have been shown to enhance the detection of small lesions with increased sensitivity and decreased interpretation time; however, increased false-positive rates have been found. ${ }^{14-17}$ Many authors have found that MIPs are of greatest benefit when used for the detection of smaller lesions $(<4$ $\mathrm{mm}$ ) because larger lesions were detected at an equivalent rate when using thin-section source images. Axial source images yield a lower false-positive rate, consistent with the results in our study, and thus a higher positive predictive value. Thus, given the importance of lesion-detection accuracy, we believe that the use of CUBE MIPs for the evaluation of brain metastases should play a 
role similar to the use of MIPs in chest imaging; MIPs can help provide a global overview of the presence of lesions, which can subsequently help focus the reader on a particular area in the brain for a more targeted assessment. Specifically, an equivocal lesion that is identified on MIPs can be cross-referenced to source images to confirm its authenticity as a true metastasis. Our study suggests that this can be achieved without significantly increasing the interpretation time.

A limitation of our study, which was also encountered by Kato et $\mathrm{al}^{8}{ }^{8}$ is the identification of false-positive lesions on CUBE images. Despite the predominant black-blood contrast of CUBE, scattered regions of short-segment vascular enhancement persist. Thus, small vessels may be difficult to differentiate from punctate enhancing metastases, and often in clinical practice, concurrent review of IR-FSPGR-BRAVO is necessary to confirm the nature of these enhancing foci by showing their continuity with vascular structures. This issue persists on MIP and may even be more problematic given the reduced ability to trace the origin of a given focus of enhancement due to thicker slabs and fewer images. Future investigation using improved blood flow suppression techniques with CUBE may help to address this dilemma. The use of MIPs in patients with innumerable $(>20)$ lesions also poses a challenge because superimposition of lesions may occur with thick-slab MIPs, thereby hampering differentiation of separate-but-adjacent metastases. Finally, the generalizability of our study results is somewhat limited because this study was performed at a single institution on $3 \mathrm{~T}$ MR imaging scanners of a single vendor type using a specific gadolinium-based contrast agent (gadobenate dimeglumine). Additional studies that include a larger sample size, more scanner types and of different magnet strength, more raters with varying levels of experience, and different contrast agents including macrocyclic agents (especially given the issue of intracranial gadolinium deposition) are needed to further validate our results. Future studies can also explore the potential use of MIPs for characterization of metastases beyond the total number of lesions, including defining tumor extent, intratumoral features, and effects of radiation treatment.

\section{CONCLUSIONS}

MIPs have been established in chest imaging for pulmonary nodule assessment and can be successfully extrapolated to brain metastatic disease. The use of oC-MIP or nC-MIP for the detection of multiple brain metastases yields reduced reading time without sacrificing diagnostic sensitivity compared with source CUBE and IR-FSPGR-BRAVO. However, the use of thick-slab oC-MIP provides higher lesion conspicuity, which can aid in overall lesion detection, especially of smaller lesions. While MIPs may not entirely replace the use of thin source images, given the limitations described in this study, they may serve as a complementary tool to enhance visualization of lesions.

Disclosures: Max Wintermark—UNRELATED: Board Membership: GE NFL Advisory Board. Greg Zaharchuk-UNRELATED: Board Membership: GE Healthcare, Comments: Research funding*. ${ }^{\star}$ Money paid to the institution.

\section{REFERENCES}

1. Lam TC, Sahgal A, Chang EL, et al. Stereotactic radiosurgery for multiple brain metastases. Expert Rev Anticancer Ther 2014;14: 1153-72 CrossRef Medline

2. Tsao MN, Lloyd NS, Wong RKS, et al; Supportive Care Guidelines Group of Cancer Care Ontario's Program in Evidence-Based Care. Radiotherapeutic management of brain metastases: a systematic review and meta-analysis. Cancer Treat Rev 2005;31:256-73 CrossRef Medline

3. Sills AK. Current treatment approaches to surgery for brain metastases. Neurosurgery 2005;57(5 Suppl):S24-32; discusssion S1-S4 Medline

4. Yamamoto M, Serizawa T, Shuto T, et al. Stereotactic radiosurgery for patients with multiple brain metastases (JLGK0901): a multiinstitutional prospective observational study. Lancet Oncol 2014;15: 387-95 CrossRef Medline

5. Serizawa T, Hirai T, Nagano O, et al. Gamma knife surgery for 1-10 brain metastases without prophylactic whole-brain radiation therapy: analysis of cases meeting the Japanese prospective multiinstitute study (JLGK0901) inclusion criteria. J Neurooncol 2010;98: 163-67 CrossRef Medline

6. Park J, Kim J, Yoo E, et al. Detection of small metastatic brain tumors: comparison of 3D contrast-enhanced whole-brain blackblood imaging and MP-RAGE imaging. Invest Radiol 2012;47: 136-41 CrossRef Medline

7. Majigsuren M, Abe T, Kageji T, et al. Comparison of brain tumor contrast-enhancement on T1-CUBE and 3D-SPGR Images. Magn Reson Med Sci 2016;15:34-40 CrossRef Medline

8. Kato Y, Higano S, Tamura H, et al. Usefulness of contrast-enhanced T1-weighted sampling perfection with application-optimized contrasts by using different flip angle evolutions in detection of small brain metastasis at $3 \mathrm{~T}$ MR imaging: comparison with magnetization-prepared rapid acquisition of gradient echo imaging. AJNR Am J Neuroradiol 2009;30:923-29 CrossRef Medline

9. Fukuoka H, Hirai T, Okuda T, et al. Comparison of the added value of contrast-enhanced 3D fluid-attenuated inversion recovery and magnetization-prepared rapid acquisition of gradient echo sequences in relation to conventional postcontrast T1-weighted images for the evaluation of leptomeningeal diseases at 3T. AJNR AmJ Neuroradiol 2010;31:868-73 CrossRef Medline

10. Komada T, Naganawa S, Ogawa H, et al. Contrast-enhanced MR imaging of metastatic brain tumor at 3 Tesla: utility of $\mathrm{T}(1)$ weighted SPACE compared with $2 \mathrm{D}$ spin echo and $3 \mathrm{D}$ gradient echo sequence. Magn Reson Med Sci 2008;7:13-21 CrossRef Medline

11. Takeda T, Takeda A, Nagaoka T, et al. Gadolinium-enhanced threedimensional magnetization-prepared rapid gradient-echo (3D MP-RAGE) imaging is superior to spin-echo imaging in delineating brain metastases. Acta Radiol 2008;49:1167-73 CrossRef Medline

12. Yoshida A, Tha KK, Fujima N, et al. Detection of brain metastases by 3-dimensional magnetic resonance imaging at $3 \mathrm{~T}$ : comparison between T1-weighted volume isotropic turbo spin echo acquisition and 3-dimensional T1-weighted fluid-attenuated inversion recovery imaging. J Comput Assist Tomogr 2013;37:84-90 CrossRef Medline

13. Kwak HS, Hwang S, Chung GH, et al. Detection of small brain metastases at $3 \mathrm{~T}$ : comparing the diagnostic performances of contrastenhanced T1-weighted SPACE, MPRAGE, and 2D FLASH imaging. Clin Imaging 2015;39:571-75 CrossRef Medline

14. Valencia R, Denecke T, Lehmkuhl L, et al. Value of axial and coronal maximum intensity projection (MIP) images in the detection of pulmonary nodules by multislice spiral CT: comparison with axial 1-mm and 5-mm slices. Eur Radiol 2006;16:325-32 CrossRef Medline

15. Kilburn-Toppin F, Arthurs OJ, Tasker AD, et al. Detection of pulmonary nodules at paediatric CT: maximum intensity projections and axial source images are complementary. Pediatr Radiol 2013;43: 820-26 CrossRef Medline

16. Jankowski A, Martinelli T, Timsit JF, et al. Pulmonary nodule detection on MDCT images: evaluation of diagnostic performance using 
thin axial images, maximum intensity projections, and computerassisted detection. Eur Radiol 2007;17:3148-56 CrossRef Medline

17. Diederich S, Lentschig MG, Overbeck TR, et al. Detection of pulmonary nodules at spiral CT: comparison of maximum intensity projection sliding slabs and single-image reporting. Eur Radiol 2001;11: 1345-50 CrossRef Medline

18. Bae YJ, Choi BS, Lee KM, et al. Efficacy of maximum intensity projection of contrast-enhanced 3D turbo-spin echo imaging with improved motion-sensitized driven-equilibrium preparation in the detection of brain metastases. Korean J Radiol 2017;18:699-709 CrossRef Medline
19. Sepulveda F, Yáñez P, Carnevale MD, et al. MIP improves detection of brain metastases. J Comput Assist Tomogr 2016;40:997-1000 CrossRef Medline

20. Peloschek P, Sailer J, Weber M, et al. Pulmonary nodules: sensitivity of maximum intensity projection versus that of volume rendering of 3D multidetector CT data. Radiology 2007;243:561-69 CrossRef Medline

21. Landis JR, Koch GG. The measurement of observer agreement for categorical data. Biometrics 1977;33:159-74 CrossRef Medline

22. Bland JM, Altman DG. Multiple significance tests: the Bonferroni method. BMJ 1995;310:170 CrossRef Medline 